\title{
eJRIEPS
}

Ejournal de la recherche sur l'intervention en éducation physique et sport

$10 \mid 2006$

Varia

\section{Vidéographie des productions motrices}

\section{Didier Barthès}

\section{OpenEdition}

\section{Journals}

Édition électronique

URL : https://journals.openedition.org/ejrieps/7174

DOI : 10.4000/ejrieps.7174

ISSN : 2105-0821

\section{Éditeur}

ELLIADD

Édition imprimée

Pagination : 16-20

Référence électronique

Didier Barthès, «Vidéographie des productions motrices », eJRIEPS [En ligne], 10 | 2006, mis en ligne le 01 juillet 2006, consulté le 29 octobre 2021. URL : http://journals.openedition.org/ejrieps/7174 ; DOI : https://doi.org/10.4000/ejrieps.7174

\section{(c) (7)}

La revue eJRIEPS est mise à disposition selon les termes de la Creative Commons Attribution 4.0 International License. 


\section{Vidéographie des productions motrices}

Didier Barthès

LEMME - Toulouse III - France

Résumé

L'approche technologique constitue le moyen d'aborder les faits naturels en les investiguant dans leur complexité en vue de produire des outils de lecture, de compréhension ou de transformation des productions. À ce titre les artefacts développés revêtent souvent un statut d'instrument cognitif facilitant, lors de leur mise en œuvre, la computation en langage naturel. En ce sens, l'approche technologique par les artefacts qu'elle permet de produire, développe le champ de connaissances qu'elle investigue.

Ce projet s'inscrit dans le cadre d'une "recherche en intervention " au sens où Bouthier (2000) la définit, renvoyant à une finalité d'optimisation de l'intervention. À ce titre le logiciel présenté dans cet article constitue un instrument de formation et de diagnostic à disposition des formateurs (enseignants, entraîneurs), aussi bien dans le cadre de l'évaluation formative que dans celui de l'expertise des productions motrices. II constitue un support dans l'analyse réflexive permettant de réduire l'écart entre le modèle ou l'analyse de l'expert et la conscience en acte chez le pratiquant.

L'approche technologique constitue le moyen d'aborder les faits naturels en les investiguant dans leur complexité en vue de produire des outils de lecture, de compréhension ou de transformation des productions, permettant ainsi d'établir les registres de technicité du domaine abordé (Bouthier D. 2000). À ce titre les artefacts développés revêtent un statut d'instrument cognitif facilitant, lors de leur mise en œuvre, la computation en langage naturel. En ce sens, l'approche technologique par les artefacts qu'elle permet de produire, développe le champ de connaissances qu'elle investigue : Les règles de production de ces connaissances ouvrent sur des perspectives d'élaboration de corpus qui en se formalisant donnent du sens aux pratiques (explicitation) et, lorsqu'elles sont formalisées, prennent un caractère scientifique propre à définir un champ autonome (Barthès D. 2000). L'approche 
technologique s'inscrit donc dans le champ de la recherche technologique qui est selon Staudenmaier (1988) l'étude des connaissances scientifiques comme disciplines outils et l'étude des outils à travers les produits de projets techniques réalisés.

Ce projet s'inscrit dans le cadre d'une "recherche en intervention " au sens où Bouthier (2000) la définit, renvoyant à une finalité d'optimisation de l'intervention. L'objet même de la recherche est constitué par la rationalisation des contenus ou des procédures de transformation du processus d'intervention dans l'enseignement de l'éducation physique et sportive ou dans l'entraînement sportif (Bouthier et Durey 1994, Bouthier 1999). La conception du projet repose sur les différents types de connaissances du système sociotechnique de production (connaissances scientifiques, technologiques, personnelles).

À ce titre le logiciel présenté constitue un instrument de formation et de diagnostic à disposition des formateurs (enseignants, entraîneurs), aussi bien dans le cadre de l'évaluation formative que dans celui de l'expertise des productions motrices ; II constitue un support dans l'analyse réflexive (Vermersch 2004) permettant de réduire l'écart entre le modèle ou l'analyse de l'expert et la conscience en acte chez le pratiquant.

\section{Méthode}

Une démonstration du logiciel VidéoGraphe est menée sur la base de différents types de productions motrices (Cyclisme, Vitesse, Gymnastique, Sports collectifs).

Le logiciel VidéoGraphe présente des caractéristiques qui font de cet artefact un instrument cognitif pertinent dans l'analyse de séquences vidéo et les formalismes graphiques associés aux images. Permettant de mener des analyses visuelles comparées, il constitue un support adapté aux besoins des formateurs par les variables qu'il permet de mettre en évidence. Le caractère de "transparence opérative » de l'artefact (Rabardel 1995) garantit pour l'enseignant l'identification et la neutralisation des effets de la transposition opérée, en facilitant l'intelligibilité et la transmission des notions en jeu dans l'interaction didactique et pédagogique.

Le module principal du logiciel, propose la lecture de séquences vidéo numériques présentées en multifenêtres indépendantes mais également synchronisables. (Module Interface et Visionnage comparatif). II fournit aussi bien les informations habituelles (durées, nombre d'images etc.) que des moyens de lecture utiles à l'analyse (réglage de la 
vitesse de lecture de 0.5 à 2 ; lecture image par image avant comme arrière); la sélection temporelle de la séquence à visionner (définition du début et de la fin de la séquence). D'autre part, il permet de capturer, à la volée éventuellement, les images de la vidéo pour une utilisation indépendante (impression, enregistrement au format image). Enfin, il permet de capturer dans une même fenêtre, une succession d'images permettant de constituer un "kinogramme » issu de la vidéo, pour une utilisation indépendante (impression, enregistrement au format image).

II s'agit donc d'un moyen de repérage et d'extraction de l'information brute des images, associé à la possibilité de comparer visuellement des productions synchronisées. A ce titre, il constitue un moyen d'investigation et d'autoscopie comparative utile in situ.

Le deuxième module propose une phénoménographie à associer aux vidéos numériques. Cet aspect du logiciel vient illustrer les variables relatives aux productions motrices identifiées comme typiques par l'utilisateur expert et à destination de l'utilisateur apprenant, en jouant sur le support graphique associé aux données vidéos. Différents outils de dessin disponibles pour l'utilisateur, permettent d'associer divers graphismes au support, en vue de la mise en évidence de caractéristiques des productions motrices. II s'agit donc d'une base de données graphiques associées aux images (Module Graphismes associés aux images). Un écran permet de visionner la vidéo sélectionnée selon les mêmes conditions que dans le premier module, et de capturer, à la volée éventuellement, une image dans un fenêtre graphique. A l'aide des outils habituels de dessin (à main levée, traits, ellipses, couleurs et épaisseur du trait, trame à superposer etc.) des graphismes divers peuvent être ajoutés à l'image capturée. Celle-ci peut être immédiatement utilisée (impression), ou bien enregistrée au format image. Par ailleurs, des informations textuelles peuvent être associées à l'image (Titre, commentaires), qui dans ce cas se trouve être indexée à la vidéo, ce qui permettra lors d'un prochain visionnage de la vidéo dans le module Graphisme, l'affichage synchronisé de l'image avec la vidéo (avec pause de lecture associée).

II s'agit donc là d'un module destiné plus particulièrement à l'expert dans l'analyse des production en vue de l'extraction et de la mise en évidence des éléments caractéristiques des productions motrices (repérage des aspects typiques, moments clefs etc.). De plus, il permet à l'expert de formaliser et d'illustrer son expertise, tant dans les aspects didactiques que pédagogiques. 


\section{Discussion.}

L'intérêt de ce type d'instrument réside dans la facilitation de la formalisation chez les enseignants des savoirs et contenus. Chez les élèves et sportifs, elle permet la comparaison immédiate de sa propre production avec d'autres plus ou moins typiques par l'intermédiaires d'une caractérisation phénoménographique illustrant l'expertise et les notions auxquelles l'enseignant, l'entraîneur fait appel dans sa démarche de formation. Cette approche constitue donc un des moyens de connaissance pour les recherches en intervention, articulant les savoirs en jeu et leurs procédures de diffusion ou de dévolution, et dont le paradigme diffère en cela significativement de la "simple " innovation. Elle porte sur les procédures liées à l'expérience et transmises en vue de transformer les représentations et habiletés des pratiquants. Cet aspect des recherches technologiques portant sur la formalisation des "techniques corporelles" constitue alors un des versants de la didactique en tant que discipline outil en milieu sportif ou scolaire. À ce titre, c'est comme le souligne Bouthier (2000), « au double titre de discipline outil, contributoire à la didactique et de discipline objet ayant en commun avec la didactique l'objet "Transmission des Techniques Corporelles » que la technologie des APSA a à voir avec les recherches didactiques ».

Cette démarche interroge donc le champ de la didactique professionnelle et déplace ainsi le point de vue de la problématique classique en Sciences de l'Éducation des didactiques des disciplines. Plutôt que de focaliser exclusivement sur les savoirs ou plus largement sur les contenus disciplinaires enjeux de formation, elle se polarise aussi sur l'analyse de l'activité de travail des enseignants confrontés à la diffusion des savoirs et la transformation des représentations chez leurs élèves. Dans cette perspective l'étude des connaissances en actes utiles pour l'action (cognition fonctionnelle située), des conditions du partage et de la structuration de l'expérience au sein de systèmes sociotechniques, est centrale : il s'agit de mettre l'élève avec ses savoirs et représentations sur le même registre notionnel que l'enseignant et ses savoirs disciplinaires. Ces concepts d'ordre pragmatique et les composantes motrices et relationnelles de la situation d'interaction didactique, sont constitutives des compétences professionnelles à développer chez l'enseignant. Elles vont pour partie inspirer les contenus et les stratégies de formation en s'appuyant sur des artefacts facilitant leur explicitation et leur structuration. Les théories et méthodologies de l'action et de la formation alors mobilisées ne le sont pas en soit et pour validation, mais pour guider et structurer les savoirs en actes, et de la même manière, définir de façon plus explicite le rôle de l'intervention dans la constitution de ces savoirs. 
Dans des recherches scientifiques en didactique où l'appropriation des techniques corporelles constitue l'un des enjeux et où il y a une forte composante expérientielle, la prise en compte des points de vue suggérés par la Technologie des APSA et la Didactique Professionnelle présente sans doute un axe de développement fécond pour le champ des activités physiques en général et celui de l'EPS en particulier. L'étude d'impact du recours à ce type de démarche constitue certainement un moyen pertinent dans l'investigation des procédures de transmission des savoirs ainsi que dans l'identification de savoirs expérientiels non formalisés. C'est là un des axes d'étude que nous développons dans le cadre d'un projet innovant (PAI06 sur l'académie de Toulouse : "CoopVidéoGraphe »), auprès d'enseignants en situation d'enseignement et utilisant ce type de démarche en tant qu'instrument d'autoscopie comparative chez leurs élèves.

\section{Bibliographie}

Barthès D. (2000). Sémiographie des sports collectifs. Approche technologique des données positionnelles des joueurs en rugby. Démonstration du logiciel Sémiographe. Communication en Atelier technologique. Colloque ARIS, Grenoble 14-16 décembre. Bouthier D. (1999). Quelles compétences pour l'intervention éducative dans les pratiques sportives ? Analyse de situations professionnelles et d'activités de formation d'entraîneurs et d'enseignants. Besançon. Conférence au Congrès de l'A.I.E.S.E.P., 7-9 avril.

Bouthier D. (2000). " l'intervention en eps, panorama des axes et des types de recherches Conduites ces dernières années ». Colloque ARIS, Grenoble 14-16 décembre.

Bouthier D., \& Durey A.(1994). La technologie des APS. In Impulsions, Paris, INRP, 95-124.

Rabardel P. (1995). Les hommes et les technologies. Approche cognitive des instruments. Paris. A. Colin.

Staudenmaier J. (1988). L'histoire des sciences et la question :'Les technologies sont-elles des sciences appliquées ?". Construire une éthique de l'enseignement scientifique. Courrier du CETHES. n5. Déc.

Vermersch P. (2004). Analyse de pratique et technique d'aide à l'explicitation. Entretiens de I'INSEP. Paris. 11mai. 